\title{
Appendix 8: Template of Supervisor's Adherence Checklist
}

\author{
(C) 2012 Mark Widdowson
}

Transactional Analysis Psychotherapy for Depression - Supervisor's Adherence Checklist

Therapist:

Supervisor:

\section{Date Completed:}

This form is for completion by the supervisor of the participating therapist and will be used for the purposes of quality control and ensuring that competent TA therapy has been delivered to the clients participating in the research.

The twelve therapeutic tasks listed below constitute the essential core treatment plan for depression. Please tick next to each item to indicate whether you feel the therapist is attending to this task in the therapy and give the therapist a rating using the six-point scale for each item. Please circle the N/A option if the item is not applicable. In the notes section, under each item and before the scale, please indicate how far the therapist achieved that item.

1 Much improvement in application needed: the therapist felt like a beginner, as if they didn't have the concept.

2 Moderate improvement needed: the therapist seemed like an advanced beginner, who is beginning to do this, but needs to work on the concept more.

3 Slight improvement in application needed: the therapist needs to make a focused effort to do more of this.

4 Adequate application of principle: the therapist did enough of this, but needs to keep working on improving how well they do it.

5 Good application of principle: the therapist did enough of this and did it skillfully.

6 Excellent application of principle: the therapist did this consistently and even applied it in a creative way.

\section{Key Therapeutic Tasks in Transactional Analysis Treatment of Depression}

1. Create an 'I'm OK- You're OK' relationship where the client feels safe enough to explore their thoughts, feelings and experiences and begin to internalise the experience of being accepted

Notes:

\begin{tabular}{|c|c|c|c|c|c|c|}
\hline $\begin{array}{l}\text { Much } \\
\text { improvment } \\
\text { needed }\end{array}$ & $\begin{array}{l}\text { Moderate } \\
\text { improvment } \\
\text { needed }\end{array}$ & $\begin{array}{l}\text { Slight } \\
\text { improvment } \\
\text { needed }\end{array}$ & Adequate & Good & Excellent & $\begin{array}{l}\text { Not } \\
\text { Applicable }\end{array}$ \\
\hline 1 & 2 & 3 & 4 & 5 & 6 & $N / A$ \\
\hline
\end{tabular}


2. Identify, reflect upon the origins of and re-evaluate self-critical ego state dialogue

Notes:

\begin{tabular}{|c|c|c|c|c|c|c|}
\hline $\begin{array}{l}\text { Much } \\
\text { improvment } \\
\text { needed }\end{array}$ & $\begin{array}{l}\text { Moderate } \\
\text { improvment } \\
\text { needed }\end{array}$ & $\begin{array}{l}\text { Slight } \\
\text { improvment } \\
\text { needed }\end{array}$ & Adequate & Good & Excellent & $\begin{array}{l}\text { Not } \\
\text { Applicable }\end{array}$ \\
\hline 1 & 2 & 3 & 4 & 5 & 6 & $\mathrm{~N} / \mathrm{A}$ \\
\hline
\end{tabular}

3. Identify, re-evaluate and challenge contaminations and script beliefs which negatively impact on the individual's self-concept and expectations of others and life

Notes:

\begin{tabular}{|c|c|c|c|c|c|c|}
\hline $\begin{array}{l}\text { Much } \\
\text { improvment } \\
\text { needed }\end{array}$ & $\begin{array}{l}\text { Moderate } \\
\text { improvment } \\
\text { needed }\end{array}$ & $\begin{array}{l}\text { Slight } \\
\text { improvment } \\
\text { needed }\end{array}$ & Adequate & Good & Excellent & $\begin{array}{l}\text { Not } \\
\text { Applicable }\end{array}$ \\
\hline 1 & 2 & 3 & 4 & 5 & 6 & $\mathrm{~N} / \mathrm{A}$ \\
\hline
\end{tabular}

4. Support the individual to recognise, re-evaluate and challenge self-limiting systems of thinking, behaviour and experience which maintain the depression (racket system)

Notes:

\begin{tabular}{|c|c|c|c|c|c|c|}
\hline $\begin{array}{l}\text { Much } \\
\text { improvment } \\
\text { needed }\end{array}$ & $\begin{array}{l}\text { Moderate } \\
\text { improvment } \\
\text { needed }\end{array}$ & $\begin{array}{l}\text { Slight } \\
\text { improvment } \\
\text { needed }\end{array}$ & Adequate & Good & Excellent & $\begin{array}{l}\text { Not } \\
\text { Applicable }\end{array}$ \\
\hline 1 & 2 & 3 & 4 & 5 & 6 & $\mathrm{~N} / \mathrm{A}$ \\
\hline
\end{tabular}

5. Explore, reflect upon and change stroking patterns (accepting positive strokes, giving self positive strokes, reduction in negative self-stroking/ self-criticism)

Notes:

\begin{tabular}{|c|c|c|c|c|c|c|}
\hline $\begin{array}{l}\text { Much } \\
\text { improvment } \\
\text { needed }\end{array}$ & $\begin{array}{l}\text { Moderate } \\
\text { improvment } \\
\text { needed }\end{array}$ & $\begin{array}{l}\text { Slight } \\
\text { improvment } \\
\text { needed }\end{array}$ & Adequate & Good & Excellent & $\begin{array}{l}\text { Not } \\
\text { Applicable }\end{array}$ \\
\hline 1 & 2 & 3 & 4 & 5 & 6 & $\mathrm{~N} / \mathrm{A}$ \\
\hline
\end{tabular}

6. Identify and challenge discounting and grandiosity (e.g 'if things go wrong it is my fault' - discounts external factors and is grandiose about role of self)

Notes:

\begin{tabular}{|c|c|c|c|c|c|c|}
\hline $\begin{array}{l}\text { Much } \\
\text { improvment } \\
\text { needed }\end{array}$ & $\begin{array}{l}\text { Moderate } \\
\text { improvment } \\
\text { needed }\end{array}$ & $\begin{array}{l}\text { Slight } \\
\text { improvment } \\
\text { needed }\end{array}$ & Adequate & Good & Excellent & $\begin{array}{l}\text { Not } \\
\text { Applicable }\end{array}$ \\
\hline 1 & 2 & 3 & 4 & 5 & 6 & $\mathrm{~N} / \mathrm{A}$ \\
\hline
\end{tabular}


7. Support the reflection upon and re-evaluation of life experiences that have contributed to a sense of worthlessness

Notes:

\begin{tabular}{l|l|l|l|l|l}
$\begin{array}{l}\text { Much } \\
\text { improvement } \\
\text { needed }\end{array}$ & $\begin{array}{l}\text { Moderate } \\
\text { improvment } \\
\text { needed }\end{array}$ & $\begin{array}{l}\text { Slight } \\
\text { improvment } \\
\text { needed }\end{array}$ & Adequate & Good & $\begin{array}{l}\text { Excellent } \\
\text { Applicable }\end{array}$ \\
\hline 1 & 2 & 3 & 4 & 5 & 6 \\
\hline
\end{tabular}

8. Support the individual to make new decisions about how they will view themselves, relate to others and engage with the world

Notes:

\begin{tabular}{|c|c|c|c|c|c|c|}
\hline $\begin{array}{l}\text { Much } \\
\text { improvment } \\
\text { needed }\end{array}$ & $\begin{array}{l}\text { Moderateimprovment } \\
\text { needed }\end{array}$ & $\begin{array}{l}\text { Slight } \\
\text { improvment } \\
\text { needed }\end{array}$ & Adequate & Good & Excellent & $\begin{array}{l}\text { Not } \\
\text { Applicable }\end{array}$ \\
\hline 1 & 2 & 3 & 4 & 5 & 6 & $N / A$ \\
\hline
\end{tabular}

9. Support the deconfusion process whereby the individual identifies, expresses and reflects upon repressed feelings (including repressed anger and working through of grief and loss)

Notes:

\begin{tabular}{|c|c|c|c|c|c|c|}
\hline $\begin{array}{l}\text { Much } \\
\text { improvment } \\
\text { needed }\end{array}$ & $\begin{array}{l}\text { Moderate } \\
\text { improvment } \\
\text { needed }\end{array}$ & $\begin{array}{l}\text { Slight } \\
\text { improvment } \\
\text { needed }\end{array}$ & Adequate & Good & Excellent & $\begin{array}{l}\text { Not } \\
\text { Applicable }\end{array}$ \\
\hline 1 & 2 & 3 & 4 & 5 & 6 & $\mathrm{~N} / \mathrm{A}$ \\
\hline
\end{tabular}

10. Support the individual to explore and experiment with new ways of relating to others which enhance self-worth

Notes:

\begin{tabular}{|c|c|c|c|c|c|c|}
\hline $\begin{array}{l}\text { Much } \\
\text { improvment } \\
\text { needed }\end{array}$ & $\begin{array}{l}\text { Moderate } \\
\text { improvment } \\
\text { needed }\end{array}$ & $\begin{array}{l}\text { Slight } \\
\text { improvment } \\
\text { needed }\end{array}$ & Adequate & Good & Excellent & $\begin{array}{l}\text { Not } \\
\text { Applicable }\end{array}$ \\
\hline 1 & 2 & 3 & 4 & 5 & 6 & $\mathrm{~N} / \mathrm{A}$ \\
\hline
\end{tabular}

11. Designing and negotiating behavioural contracts such as awareness exercises homework, self-care contracts, exercise, diet and sleep hygiene contracts.

Notes:

\begin{tabular}{|c|c|c|c|c|c|c|}
\hline $\begin{array}{l}\text { Much } \\
\text { improvment } \\
\text { needed }\end{array}$ & $\begin{array}{l}\text { Moderate } \\
\text { improvment } \\
\text { needed }\end{array}$ & $\begin{array}{l}\text { Slight } \\
\text { improvment } \\
\text { needed }\end{array}$ & Adequate & Good & Excellent & $\begin{array}{l}\text { Not } \\
\text { Applicable }\end{array}$ \\
\hline 1 & 2 & 3 & 4 & 5 & 6 & $\mathrm{~N} / \mathrm{A}$ \\
\hline
\end{tabular}


12. Facilitate the client's attachment to and engagement with life, others and the world

Notes:

\begin{tabular}{l|l|l|l|l|l}
$\begin{array}{l}\text { Much } \\
\text { improvment }\end{array}$ & $\begin{array}{l}\text { Moderate } \\
\text { improvment }\end{array}$ & $\begin{array}{l}\text { Slight } \\
\text { improvment } \\
\text { needed }\end{array}$ & Adequate & Good & Excellent \\
\hline 1 & 2 & 3 & 4 & 5 & 6 \\
\hline
\end{tabular}

Comments on how the therapist has managed transference and countertransference with this client: 